\title{
THE LONG-TERM REACTION OF BONE TO SELF-CURING ACRYLIC CEMENT
}

\author{
J. Charnley, F. M. Follacci and B. T. Hammond, Wrightington, England \\ From the Centre for Hip Surgery, Wrightington Hospital
}

Self-curing acrylic cement is being used more and more in orthopaedic surgery to bond a weight-bearing prosthesis to bone, and in the future it may also play an important part in the treatment of certain difficult fractures. Until now we have reserved its use to elderly patients, but it gives such promise of mechanical adaptability that it would be a great step forward if it could be used also in young patients.

In a previous report (Charnley 1964) the impression was given that osteoporosis could follow the use of this cement, but the follow-up in that study was not long enough to establish whether or not osteoporosis was progressive. The present study has been directed particularly towards answering this question.

\section{CLINICAL MATERIAL}

This is mainly a radiological study of the reaction of the bone of the upper end of the femur to acrylic cement used for the fixation of femoral endoprostheses. It is a clinical study also, because it is obviously important to correlate radiological appearances with the quality of the surgical result, and to take into account any complications such as wound infection which could explain unusual radiological pictures. The study has been confined to patients who have had recent radiographs; that is, within the last year (1967).

We have tried to find as many cases as possible in which acrylic cement has been in contact with bone for over six years, but since most of the patients operated on nine years ago were already past their middle seventies, there has been a heavy loss to the series by death from natural causes. It seems, for example, that not one of the senile patients operated on during 1958 and 1959, who formed the basis of the original paper (Charnley 1960), has survived; the examples from this era which are included in this study are specimens acquired after the death of the patients during the last two years.

All the early cases were of simple femoral head prostheses inserted for femoral neck fractures. The geriatric service to the local community (Davyhulme Hospital, Manchester) is such that we have every reason to believe that we would have been told of any serious complications relating to the hip had they occurred. Between November 1962 and November 1963 most of the patients were those subjected to total prosthetic replacement of the hip (" low-friction arthroplasty") with a socket of high density polyethylene and a stainless steel femoral head.

We have deliberately excluded patients who had been operated on before November 1962 with Teflon (polytetrafluorethylene), because in finely abraded form this plastic can produce extensive erosion of bone and render impossible any interpretation of bone reactions to acrylic cement. For this reason few of the patients in the paper of 1964 reappear in the present study, and those who do reappear are those with the simple femoral head replacements.

Of 312 case records examined, 122 hips had to be discarded for the following reasons: seven because of frank infection necessitating removal of the prosthesis; twenty-three because of death; and ninety-two for failure to obtain an up-to-date radiograph of patients living at a distance or too frail to travel. This left 190 femora available for study in 174 fully documented patients, comprising forty-four femoral head prostheses and 146 total hip prostheses. The average follow-up was four years (minimum three years, maximum eight years) (Table I). The distribution of ages of patients is shown in Table II. 
The conditions for which the operations were performed were: primary osteoarthritis 114 cases; rheumatoid arthritis forty cases; ankylosing spondylitis seven cases; osteoarthritis secondary to dysplasia four cases; fracture of femoral neck or complications seventeen cases; failure of previous arthroplasty six cases; quadrantic necrosis of the head one case; secondary to infective arthritis one case.

TABLE I

DURATION OF OBSERVATION

\begin{tabular}{|l|l|l|l|l|l|l|l|l|l|l|l|}
\hline Years .. & 8 & 7 & 6.5 & 6 & 5.5 & 5 & 4.5 & 4 & 3.5 & 3 \\
\hline Number of femora & 1 & 5 & 1 & 2 & 6 & 14 & 33 & 89 & 37 & 2 \\
\hline
\end{tabular}

TABLE II

Age at Operation

\begin{tabular}{|l|c|c|c|c|c|c|c|c|c|}
\hline Age (years) . & $20-30$ & $31-40$ & $41-50$ & $51-60$ & $61-70$ & $71-80$ & $81-90$ \\
\hline Number of patients & 2 & 2 & 15 & 32 & 64 & 58 & 1 \\
\hline
\end{tabular}

\section{METHOD OF STUDY}

Most patients had radiographs at yearly intervals, with a radiograph also after the first six months; all had radiographs taken immediately before and after operation.

The radiographs were displayed simultaneously on a large illuminated surface and the quality of the bone of the femoral cortex was compared, at first by general inspection at a distance, and then more closely and by direct measurement on the radiograph. By arranging the radiographs chronologically, consistent trends towards osteoporosis or towards sclerosis could be distinguished. Sporadic appearances not in line with the general trend could be ignored if these were attributable to marked alterations in the penetrations of the $x$-rays at one of the yearly reviews. The radiological features studied included: 1) the texture of the bone of the cortex and medulla; 2) the outline or silhouette of the external surface of the cortex; 3) periosteal new bone formation; 4) bone resorption or destruction; 5) thickness of the cortical bone; 6) resorption of the stump of the femoral neck.

In attempting to appreciate osteoporosis, reliance was placed on measurements of the thickness of the cortex rather than on any general appearance of osteoporosis. The thickness of the cortex was judged in proportion to the total width of the shaft of the femur at a chosen level. This level has been taken at $\mathbf{4 0}$ millimetres above the tip of the stem of the prosthesis as measured directly on the radiograph. The thicknesses of both cortices were measured directly on the film and their sum expressed as a percentage of the external diameter of the femoral shaft at this level. An augmentation in the percentage thus represents hypertrophy and a diminution atrophy.

The accuracy with which this comparison can be made has been estimated at \pm 5 per cent because an error of 0.5 millimetre can be incurred at each of the three measurements involved. Only variations greater than 5 per cent have therefore been considered significant. It is interesting that it required a variation beyond 5 per cent to give a clear impression of atrophy or hypertrophy.

\section{RESULTS}

Preservation of cortical thickness-In 154 hips (81 per cent) the bone of the femoral shaft has remained normal; that is to say the external silhouette or profile of the cortex, the thickness of the cortical bone and the texture of the cortical bone have all remained unchanged. The average follow-up in this group is four years and two months (Table III).

VOl. 50 B, NO. 4, NOVEMBER 1968 
Two features encountered in this series are worth reporting. Firstly, eight of the nine patients followed up for more than six years fell into this group with normal cortical bone. An example of a seven-year result is illustrated in Figure 1. This patient, aged seventy, had sustained a fracture of the neck of the femur which had failed to unite after internal fixation

TABLE III

Change in Thickness of Cortex after Operation

(154 hips in which the change was insignificant)

\begin{tabular}{|c|c|c|c|}
\hline & $\begin{array}{l}\text { Reduced by } \\
1-5 \text { per cent }\end{array}$ & Unchanged & $\begin{array}{l}\text { Increased by } \\
1-5 \text { per cent }\end{array}$ \\
\hline Number of hips & 58 & 46 & 50 \\
\hline
\end{tabular}

with a nail, and two years later a prosthesis was bonded in with cement. The clinical result is good. Secondly, eleven out of the twelve hips in which a femoral head arthroplasty, using cement, had been later converted into a total arthroplasty were found to have normal bone. In these cases the bone of the femoral cortex had been exposed twice to the reaction of polymerisation without ill-effect. In these cases it was the second insertion of cement which was the subject of the four-year follow-up.

Increase or reduction of cortical thickness-In fourteen cases $(7 \cdot 3$ per cent) a significant change in thickness of the femoral cortex occurred. The external silhouette or profile of the cortex and the texture of the bone remained normal in this group (Table IV).

Hypertrophy of the cortex (on the endosteal aspect) occurred in five cases ( 2.6 per cent). The average follow-up in this group was three years and four months (maximum four years, minimum three years). On average this hypertrophy started to become apparent twenty months after the operation; in four cases it reached a maximum in sixteen months.

Four of the patients with hypertrophy had osteo-

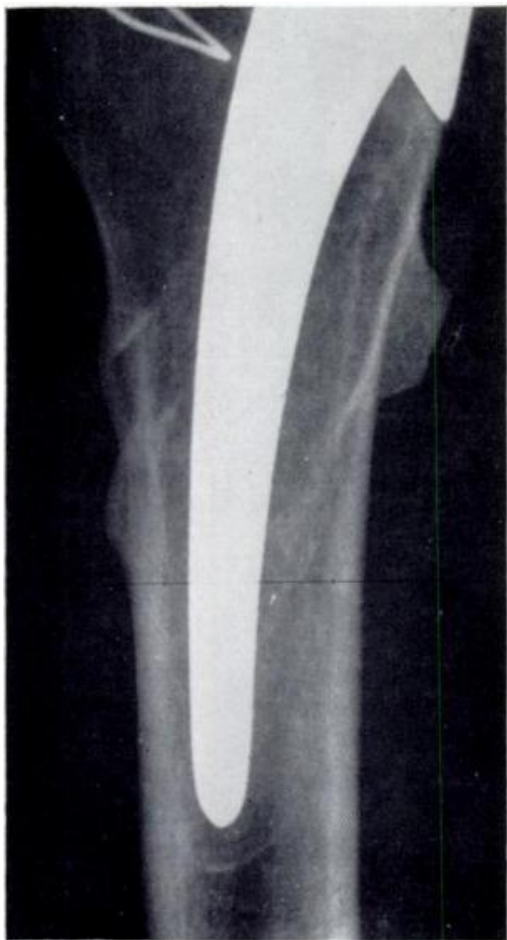

FIG. 1 porotic femora before the operation in consequence of rheumatoid arthritis or ankylosing spondylitis, and even with crutches were unable to walk at all before the operation. After the operation their function improved to the point that they could walk without pain and with minimal assistance from a stick or sticks (Figs. 2 and 3). It is tempting to attribute this improvement to the physiological response of bone to increased mechanical stress. Although this is probably the explanation the problem may not be quite so simple; many patients with atrophy of the femoral cortex before operation have had a dramatic restoration of function by the operation without any significant change in the thickness of the cortex. In one patient with bilateral hip arthroplasties, performed in the presence of atrophic bone,

Fig. 1

Typical appearance in elderly patient with prosthesis cemented in seven years previously. The track of a nail inserted earlier for femoral neck fracture is visible. The cement here is radiolucent. A faint line of condensation in the cancellous bone at the lower pole of the prosthesis outlines cement. 
there was hypertrophy of the cortex on one side but no change in thickness of the cortex on the other, despite equally satisfactory restoration of function on both sides.

Atrophy of the cortex occurred in nine cases ( 4.7 per cent). The average follow-up in this group was four years and three months (six years maximum, three and a half years minimum).

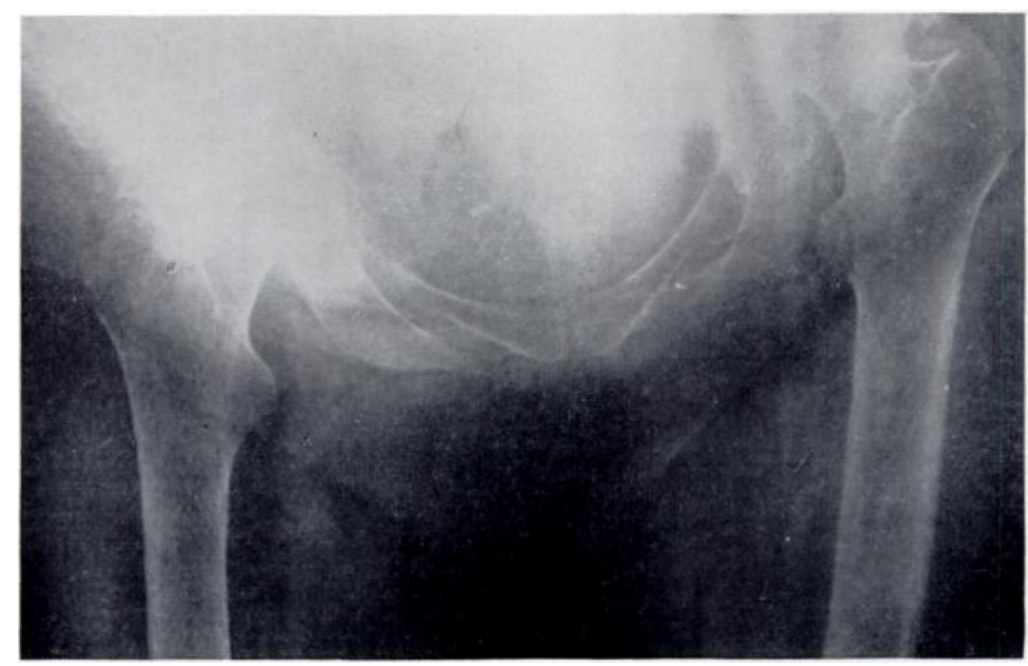

Fig. 2

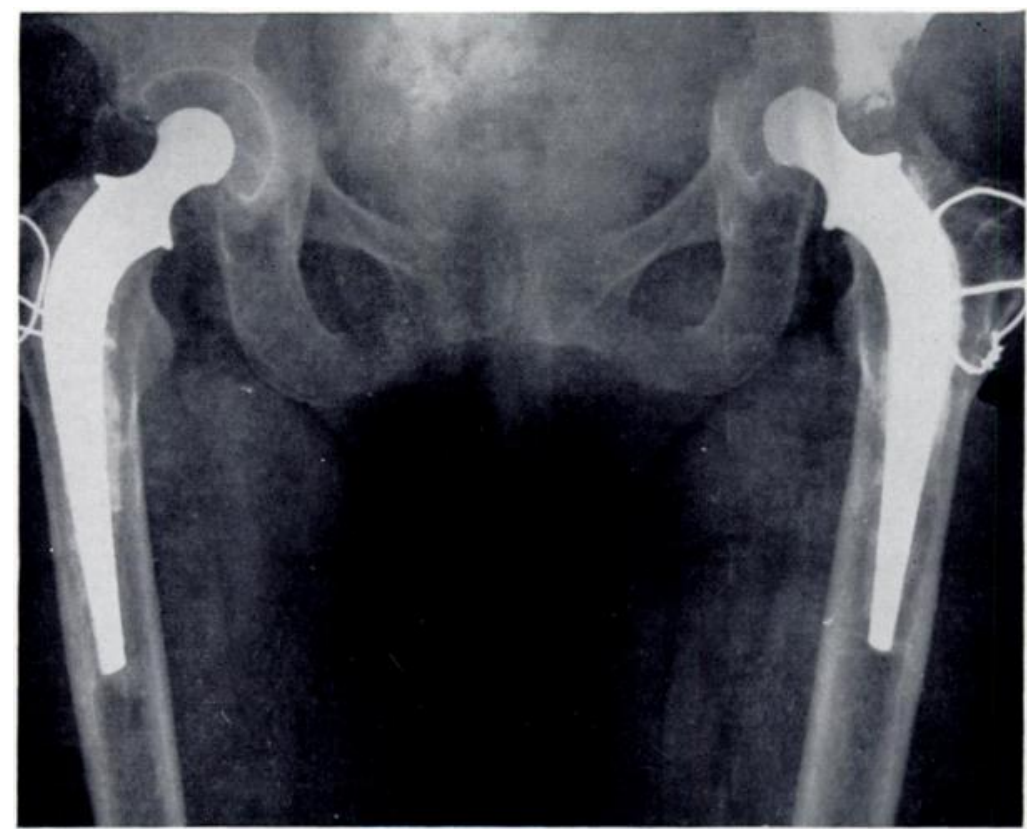

FIG. 3

Figure 2-Bilateral rheumatoid arthritis of hips with osteoporosis before operation. Figure 3-Same case four years after bilateral low friction arthroplasty. Improvement in thickness of femoral cortices. The cement is radiopaque.

In three patients, all with good clinical results, appearances suggested that the atrophy occurred continuously at about 2-3 per cent per year (that is, below the limit of accuracy of the estimation) but none of these three cases showed as much as 10 per cent in the end.

VOL. $50 \mathrm{~B}$, NO. 4, NOVEMBER 1968 
TABLE IV

Change in Thickness of Cortex after Operation

(Fourteen hips in which the change was significant)

\begin{tabular}{|c|c|c|c|c|c|c|}
\hline & $\begin{array}{c}\text { Reduced by } \\
\text { 16-20 per cent }\end{array}$ & $\begin{array}{c}\text { Reduced by } \\
\text { 11-15 per cent }\end{array}$ & $\begin{array}{c}\text { Reduced by } \\
\text { 6-10 per cent }\end{array}$ & $\begin{array}{c}\text { Increased by } \\
\text { 6-10 per cent }\end{array}$ & $\begin{array}{c}\text { Increased by } \\
\text { 11-15 per cent }\end{array}$ & $\begin{array}{c}\text { Increased by } \\
\text { 16-20 per cent }\end{array}$ \\
\hline Number of hips & 1 & 1 & 7 & 2 & 2 & 1 \\
\hline & & Atrophy & & & Hypertrophy \\
\hline
\end{tabular}

In the six remaining cases the atrophy reached a maximum in an average of eleven months and did not progress thereafter. The general clinical result in these six cases was not good, but this was because of a defective hip on the opposite side, the arthroplasty itself being successful. External hypertrophy of cortex-In eighteen cases $(9.4$ per cent) hypertrophy of the cortex occurred with alteration in the external silhouette or profile of the cortex. The texture of the bone remained normal in these cases. This type of hypertrophy results in a spindle-shaped profile illustrated in Figure 4. The centre of the spindle lies in relation to the distal part of

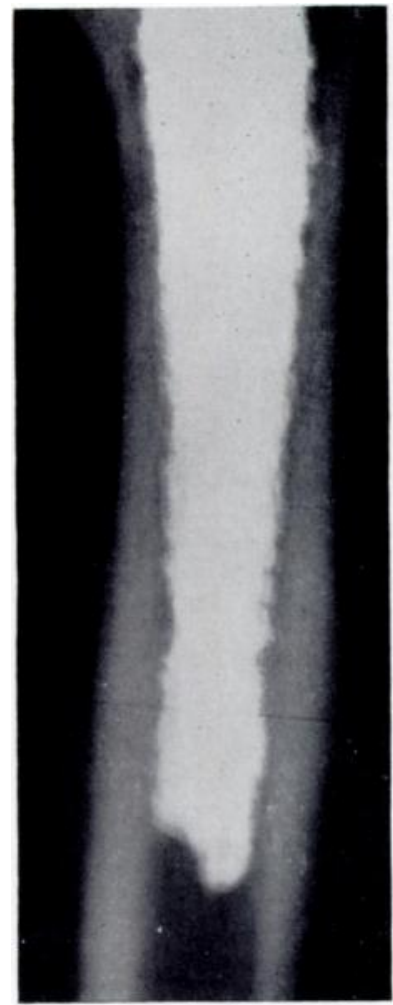

FIG. 4

Fusiform thickening of shaft of femur ascribed to increased stress at junction between flexible and artificially stiffened sections of femur. the stem of the prosthesis. In sixteen cases the level of the centre of the spindle corresponded to a point about eighteen millimetres proximal to the tip of the prosthesis. In one case the centre of the spindle lay at the level of the tip of the prosthesis, and in one case ten millimetres distal to it. On the average this spindle-shaped thickening appeared two years and two months after the operation (maximum three and a half years, minimum one year). The percentage thickness of the external diameter of the cortex, expressed in terms of the external diameter of the opposite femur at the same level, gave an average increase of 13 per cent (maximum 28 per cent; minimum 7 per cent).

This type of spindle-shaped thickening involved both cortices in eight cases, the lateral cortex only in six cases, and the medial cortex only in four cases. In all these patients function was good and there was no pain.

It seems probable that this type of spindle-shaped thickening is a physiological response to increased mechanical stress in the bone of the cortex caused by the transition from the normal, flexible shaft of the femur to the part rendered rigid by the cement and the prosthesis.

It is interesting that in no case in this study, with an average follow-up of four years, has a fracture of the shaft of the femur from trivial violence been encountered. This absence of fracture (which applies also to a much larger series of cemented prostheses not included in this series) appears to contrast with general experience with femoral prostheses used without cement.

Alteration of texture of cortex-In four cases $(2 \cdot 2$ per cent $)$ changes in the texture of the cortical bone were encountered (Figs. 5 to 8 ) which at first glance would suggest some form of chronic osteitis as the most likely cause. Three of these examples (Figs. 5 to 7) were discovered at routine radiological examination without clinical signs or symptoms to suggest local infection. The clinical result in these three cases is still completely successful four years after operation. 
The case illustrated in Figure 8 is not a complete clinical success. The patient has complained of intermittent aching pain during the whole period of four years since the operation, though he is able to walk without the support of a stick, has an excellent range of movement and presents no palpable thickening or other local signs of inflammation. We consider that there is almost certainly a chronic non-suppurative infection in this case.

In one of the three cases in which there is thickening of the cortex (Fig. 5) the thickening is clearly caused by periosteal new bone; the line of the original cortex is still visible.

In the case in which there was no thickening of the cortex (Fig. 7) there was a destructive endosteal lesion. This appearance has been present for the last three of the four post-operative years and it is not yet certain whether it is extending. An identical appearance has been encountered in another patient in a more recent series, from which a bacillus proteus was cultured.

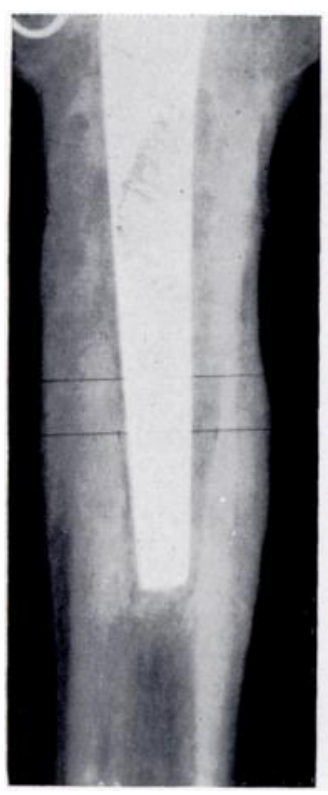

Fig. 5

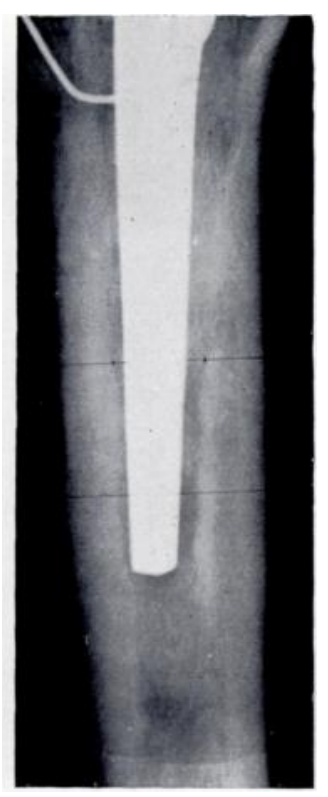

Fig. 6

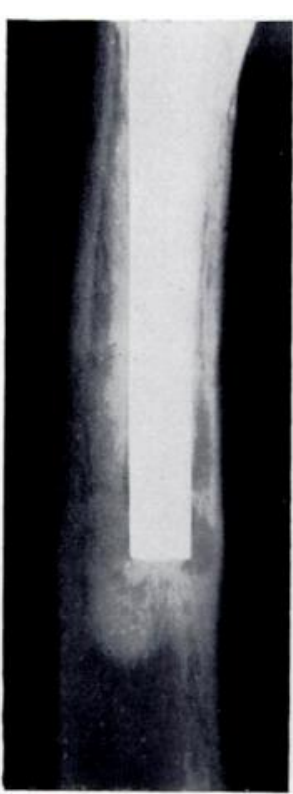

FIG. 7

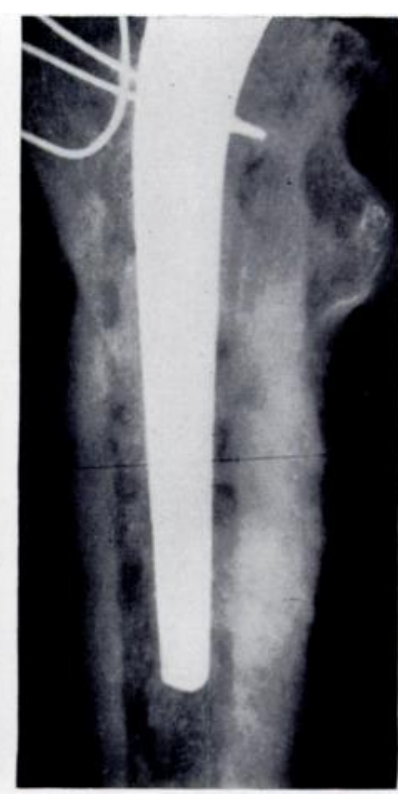

Fig. 8

Alteration of texture of cortex. Figure 5-Changed texture of cortex with periosteal new bone four years after operation, suggesting chronic non-suppurative infection. The patient was symptom-free. Figure $6-$ Changed texture of cortex, with cortical thickening four years after operation, suggesting chronic infection. The patient was symptom-free. Figure 7-Destructive lesion on endosteal surface of cortex four years after operation. The patient was symptom-free. The changes were thought to be caused by chronic infection. Figure 8 Hypertrophy of cortex with changed radiological texture four years after second operation. Cement was used in both operations. The changes are almost certainly caused by chronic infection. The patient has pain.

An appearance of sclerosis like that shown in Figure 6 was encountered in two other patients from a more recent series. Hip arthroplasty was performed on both sides but the texture of the opposite femur has remained normal for three years. The sclerotic changes on the abnormal side started within a year of the operation. The changes are associated with slight discomfort and with slight impairment of weight-bearing function. These observations suggest that the sclerosis cannot be an allergic response to the cement but that it is a chronic non-suppurative bacterial lesion.

It must be emphasised that nothing in the course after operation in these four cases indicated wound infection. If this had been so the etiology of these appearances would not be in any way obscure. All cases of known infection (seven) were discarded from the series in the original selection.

Condensation of bone adjacent to cement - In eighty-six cases ( 44.8 per cent) some condensation of cancellous bone demarcating the external surface of the cement was observed. This 
appearance shows first between one and two years after the operation; thereafter it does not seem to increase significantly. It is rarely thicker than one millimetre measured directly on the radiograph. Most of the outline is related to the distal thirty or forty millimetres of the cement (Fig. 9).

There have been opportunities to explore femora presenting this appearance, as for instance in the patient represented in Figure 10 who had pain after the insertion of a simple femoral head prosthesis. At exploratory operation the prosthesis was found still to be quite rigidly fixed in the femur, and ulceration of the acetabulum with exposure of bare bone was clearly the cause of pain (proved by the absence of pain after replacing the acetabulum with a total prosthesis).

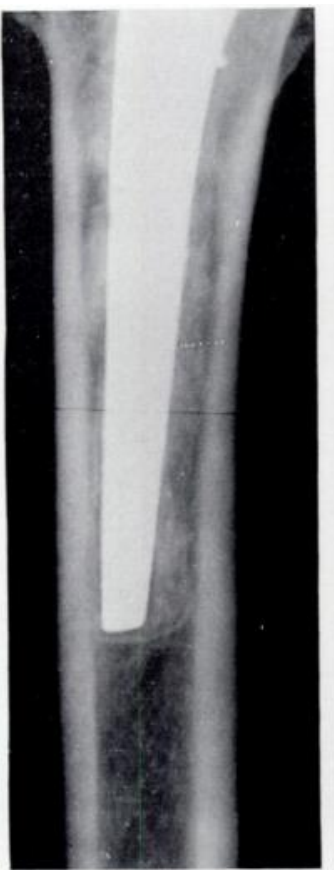

Fig. 9

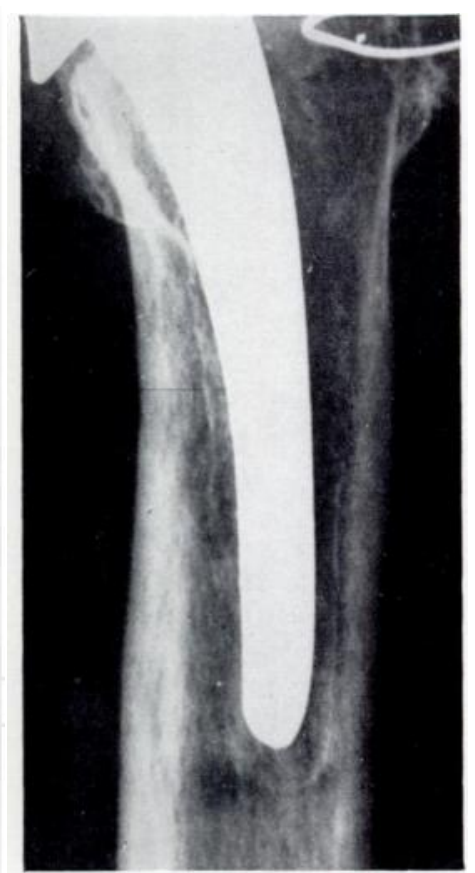

Fig. 10

Figure 9-Line of bone condensation in relation to surface of cement near tip of prosthesis. Line not visible in proximal part. Not an indication of loosening. Figure 10-Typical appearance of line of condensation of cancellous bone in contact with cement. Exploration showed that the prosthesis was rigidly fixed in the bone.

The appearance of a line of condensation between the surface of the cement and adjacent cancellous bone in itself does not indicate loosening of the prosthesis, especially when the condensation is confined only to parts of the outline of the cement. In rare cases in which a cemented prosthesis has become loose (not encountered in this series) the line of condensation in the cancellous bone has been continuous along the whole surface of the cement from the tip of the prosthesis to the trochanter.

Resorption of the femoral neck-Resorption of the femoral neck was detectable in seventy-one cases $(37.2$ per cent). This resorption averaged three millimetres, the maximum being ten millimetres. It is believed that the cause of this absorption is destruction of the blood supply by the section through the neck; this interrupts the longitudinal circulation in the Haversian canals of the cortical bone of the calcar femorale and the bone is further deprived of blood by the stripping of the periosteum from the calcar femorale during dislocation of the hip. 
Absorption of the femoral neck demonstrates that the cement is in fact transmitting load directly to the cortex of the shaft of the femur through the stem of the prosthesis. Absorption of bone cannot manifest itself by retraction from the collar of the prosthesis when the prosthesis has not been cemented, because then the prosthesis sinks into the shaft.

\section{CONCLUSIONS}

We consider that this study of nearly 200 femora with self-curing acrylic cement in the medullary canal is fully representative of the state of affairs four years after insertion of the prosthesis.

It will be noted that in only four patients $(2 \cdot 2$ per cent) have radiological changes been encountered which are in any way disquieting as regards the ultimate fate of the bone. In only one of these are there symptoms which suggest that future trouble may lie ahead. We feel that chronic osteitis from mild infection, acquired either at operation or later from the blood, is the most likely explanation of these appearances. We do not consider that these appearances result from chemical or allergic reaction to the cement because identical changes have twice been observed unilaterally in patients in whom cement has been used on both sides.

Just how long a cautious surgeon must remain apprehensive before the use of self-curing cement can be judged to be completely harmless in the human subject seems, in the light of this study, to be a matter of personal opinion. From general experience with foreign bodies of any material used as implants in the human subject, one would imagine that an average period of four years would be sufficient to enable us to pronounce this procedure safe, especially when no signs of intolerance have been encountered in any of a small sample at seven or eight years. Certainly it can be considered safe in patients whose pre-operative disability is great, and for whom orthodox techniques have little or nothing to offer.

\section{SUMMARY}

1. A study is reported of 190 femora in 174 patients in whom self-curing acrylic cement had been present in the medullary cavity of the upper end of the femur for the fixation of an endoprosthesis for an average period of four years.

2. The bone remained radiologically normal in 81 per cent of cases.

3. Improvement in the thickness of the cortex from pre-existing atrophy was noted in $\mathbf{2 \cdot 6}$ per cent.

4. In 4.7 per cent the bone showed some atrophy after insertion of the cement. This exceeded 10 per cent in only two cases. All were originally osteoporotic from polyarthritis; all were satisfactory as regards the arthroplasty itself, and the atrophy could usually be explained by disuse resulting from the state of the opposite lower extremity, or the knee on the same side. 5. In 9.4 per cent there was fusiform hypertrophy of the femoral cortex, the bony texture remaining normal. This appearance was considered physiological and benign.

6. In $2 \cdot 2$ per cent there were changes for which the most likely explanation is chronic nonsuppurative osteitis, though no collateral evidence of infection was found.

7. In 44.8 per cent there was a thin line of condensation in the cancellous bone demarcating the outer limits of the cement. This is considered to be physiological and not to indicate failure of immobilisation.

8. In 37.2 per cent there was slight resorption of the cut surface of the calcar femorale. This is considered to be physiological and to confirm the efficacy of weight transmission by cement lower down in the medullary cavity.

\section{REFERENCES}

Charnley, J. (1960): Anchorage of the Femoral Head Prosthesis to the Shaft of the Femur. Journal of Bone and Joint Surgery, 42-B, 28.

Charnley, J. (1964): The Bonding of Prostheses to Bone by Cement. Journal of Bone and Joint Surgery, 46-B, 518.

VOL. $50 \mathrm{~B}$, NO. 4, NOVEMBER 1968 